\title{
Pharmacovigilance in the vaccination program against coronavirus disease 19
}

\author{
Octavio Amancio-Chassin* \\ Pharmacovigilance Service, Centro Institucional de Farmacovigilancia, Hospital General de México Dr. Eduardo Liceaga, Mexico City, Mexico
}

Emerging and reemerging infectious viral diseases have threatened humanity throughout history. Several intertwined and synergistic factors, including demographic trends, high-density urbanization, roads, which favor high mobility of people in all modes of transport, large concentrations, altered human behaviors, environmental changes with the modification of ecosystems, and the inadequate processes of the global public health system, have accelerated the appearance and spread of viruses from other animals, as a threat to the human population.

Immunization through vaccines is a successful preventive measure in terms of health and has saved millions of lives each year. Vaccines lower the risk of disease by stimulating the body's natural defenses to provide protection. At present, we have vaccines to prevent more than 20 life-threatening diseases; this causes the life expectancy of people of all ages to increase and be healthier. Vaccination prevents 2-3 million deaths each year from diseases such as diphtheria, polio, tetanus, pertussis, influenza, hepatitis $B$, and measles. Vaccination is a key component of primary health care and an indisputable human right. Furthermore, it is one of the best investments in health for the progress of humanity.

Before starting any study in humans, it is necessary to comply with several principles and tests that clearly demonstrate the evidence on the efficacy and safety of the vaccine in animals, with negative tests of pyrogenicity, mutagenesis, and oncogenicity, among other tests; in addition, ensure that the vaccine lots for clinical studies are comparable to the non-clinical lot used in the preclinical phase and that the vaccine was prepared following good manufacturing practices, in which the purity, production procedure, and potency studies are highlighted. On the other hand, a research protocol must be developed, which is approved by the Research Ethics, Research, and Biosafety Committees of the institutions that participate in the clinical study of the vaccine.

Vaccines are the cornerstone in managing the infectious disease outbreak and are the surest means of defusing the risk of a pandemic and epidemic. The faster a vaccine is implemented, the faster an outbreak can be controlled. In May 2020, the $42^{\text {nd }}$ Global Advisory Committee on Vaccine Safety addressed the preparation of pharmacovigilance for the launch of future coronavirus disease (COVID-19) vaccines. One of their recommendations was that COVID-19 vaccine safety surveillance infrastructure and capacity should be in place in all countries and in place before the vaccine is introduced. The World Health Organization (WHO) COVID-19 vaccine safety surveillance manual specifies the need for post-authorization pharmacovigilance studies; as well as the procedure for the notification and follow-up of adverse events after immunization, adverse events of special interest, data management systems, and safety communication. The implementation of this surveillance requires local, national, regional, and global collaboration. While countries should include COVID-19 vaccine safety preparedness plans in their overall plans, when approving

\section{Correspondence:}

*Octavio Amancio-Chassin

E-mail: octavioa28@gmail.com
Date of reception: 15-06-2021

Date of acceptance: 17-06-2021

DOI: 10.24875/HGMX.M21000041
Available online: 29-07-2021

Rev Med Hosp Gen Mex. 2021;84(3):92-95 www.hospitalgeneral.mx NC-ND license (http://creativecommons.org/licenses/by-nc-nd/4.0/). 
the vaccine, these should be supported by the WHO guidelines and it is imperative that the COVID-19 vaccines initiative global access (COVAX) (coordinated by CEPI, Gavi, the Vaccine Alliance, and WHO) participate in the implementation of the vaccination program, with technical and training support, adapted to the environment of each country. A critical element of this surveillance is the length of the observation period ${ }^{1}$.

Clinical research is carried out on healthy research subjects, that is, individuals who, according to the available information, do not suffer from any significant disease relevant to the proposed study, whose body proportions and weight are within normal limits and have a mental state that allows you to understand and give valid consent for your participation in the study. If the research study requires minors, the consent of both parents must be requested; also, special populations can be evaluated, for example, pregnant women, patients with immunodeficiency or cancer diseases with chemotherapy.

Safety tests during the clinical development of vaccines consist of identifying the incidence of adverse reactions (local and systemic) that occur with the administration or application of the vaccine, among them we find erythema, inflammation, edema, pain, and decreased mobility in the limb where the vaccine is applied or systemic reactions such as decreased appetite, sleep, irritability, fever, asthenia, adynamia, hypersensitivity reactions, or symptoms of the different devices or systems that may be affected by administration of the vaccine; in addition, alterations in laboratory tests: hematic biometry, blood chemistry, liver function, serum electrolytes, coagulation tests, pancreatic enzymes, and general urine examination. Safety studies analyze all the components of the biological product, to determine which of them are responsible for producing adverse reactions: the active biological principle, adjuvants, preservatives, or stabilizers. The presence of adverse reactions is evaluated immediately (first $30 \mathrm{~min}-24 \mathrm{~h}$ after administration) and subsequently $3,7,15,30,45$, and 60 days; the observation time depends on the type of vaccine and the study population.

The regulatory authority evaluates the scientific and clinical evidence provided by the vaccine manufacturers, who, by legal obligation, must comply with certain norms regarding the presentation of data. In addition, the authorities supervise clinical studies and manufacturing practices. After insightful evaluation, data from clinical trials on vaccines are made available; either in its entirety or summarized. Regulatory authorities exhaustively evaluate the safety, efficacy, and pharmaceutical quality of each vaccine to determine if it is authorized for use. To that end, evidence and information on manufacturing are studied to understand the benefits of candidate vaccines and the risks to which individuals are exposed².

When a vaccine is approved by the country's health authority, in the case of Mexico, the Federal Commission for the Protection against Sanitary Risks (COFEPRIS), the commercialization of the vaccine implies its permanent evaluation to identify the effectiveness of the vaccine and the detection of adverse reactions or new reactions; especially late onset, in open population. Pharmacovigilance and pharmacoeconomic studies (minimization of costs, cost-effectiveness, cost utility, and cost benefit) are the tools used in Phase 4 to evaluate the safety, effectiveness, and usefulness of the biological product under real conditions of use.

Pharmacovigilance encompasses activities related to the detection, evaluation, understanding and prevention of adverse events, suspected adverse reactions, adverse reactions, events presumably attributable to vaccination or immunization, or any other safety problem related to the use of drugs and vaccines ${ }^{3}$. In other words, the fundamentals of pharmacovigilance are to identify the safety of drugs and vaccines in the medical field and the typification of new unknown adverse reactions at the time of approval of the various products (medicines, biotech, and vaccines) on the market.

In the case of vaccines, safety is evaluated through the events presumably attributable to vaccination or immunization; which are defined as the clinical manifestations or medical event that occur after vaccination and are supposedly attributed to vaccination or immunization, the timing will depend on each of the vaccines ${ }^{3}$.

At present, there are eight types of vaccines against COVID-19, which were presented to COFEPRIS, five of them obtained their emergency approval, to be administered, in the Mexican population":

1. BNT162b2 (mRNA) from Pfizer Inc./BioNtech, approved on November 11, 2020

2.AZD1222 (adenovirus) from Astra Zeneca/Oxford University, approved on January 4, 2021

3. Sputnik V (Non-replicating viral vector) from Instituto Gamaleya, approved on February 9, 2021

4. Ad-nCoV (Non-replicating Viral Vector) from CanSino Biologics Inc., approved on February 9, 2021

5. CoronaVac (inactive virus) from Sinovac Research and Development Co., approved on February 9, 2021

6. Ad26.CoV 2.5 (Adenovirus) from Janssen/Johnson and Johnson, Phase III investigation protocol 
7. CVnCoV (mRNA) from CureVac, Phase III research protocol

8. NVX CoV 2373 (protein subunit) from Novavax Inc., Phase III research protocol

After its emergency approval, it is necessary to implement pharmacovigilance studies (whose objective is safety), which is defined as any clinical study related to an authorized drug or vaccine, which seeks to identify, characterize, or quantify a safety risk, which can confirm the safety profile of the drug or vaccine, propose effective measures, and measure their effectiveness in minimizing risks.

In Mexico, 26,422,830 people have been vaccinated from December 24, 2020, to June 14, 2021, in its different stages, of them $15,175,689$ individuals with a complete schedule and 11,247,141 at least one dose. The total of doses applied is $37,422,800$ of the different vaccines approved by COFEPRIS ${ }^{5}$.

Until June 12, 2021, 21,587 events supposedly attributable to vaccination or immunization have been reported, of which 447 (2.0\%) were serious. Regarding gender, $194(43.4 \%)$ subjects were male and $253(56.6 \%)$ were female. Table 1 shows the total number of events supposedly attributable to vaccination or immunization and the number of serious events, for each type of vaccine authorized in Mexico ${ }^{6}$.

It is very important that health professionals not only fervently report all adverse events reported by patients but also instruct vaccinated people to immediately inform a health professional or health authorities of the presence of any event. By reporting all relevant adverse events, regulatory authorities can better assess whether they are caused by the vaccine and can help detect potential risk problems caused by licensed vaccines (Table 1).

As part of the examination and surveillance of all suspected cases of vaccine adverse events, regulatory authorities should establish the incidence of events presumably attributable to vaccination or immunization, especially serious events (for example, anaphylaxis) or those that are related to some risk factor (age, sex, obesity, arterial hypertension, diabetes mellitus, etc.), it is important to monitor even though there is no evidence that they are produced by the effect of the vaccine. It is important to have information on the rates of these events that could occur in unvaccinated people, to quickly detect any increase so that it can be thoroughly investigated by the regulatory authorities. The main limitation in the current scenario is time constraints; therefore, the urgency for a COVID-19 vaccine to be licensed is concerning and marks a fine line between the speed, safety, and efficacy of the vaccine.
Table 1. Total number of events supposedly attributable to vaccination or immunization and the number of serious events, for each type of vaccine authorized in Mexico

\begin{tabular}{|l|c|c|}
\hline $\begin{array}{l}\text { Type of vaccine } \\
\text { authorized }\end{array}$ & $\begin{array}{c}\text { No. of events presumably attributable to } \\
\text { vaccination }\end{array}$ \\
\hline & Total & Severe \\
\hline Pfizer-BioNtech & 16,095 & 194 \\
\hline Astra Zeneca & 2805 & 103 \\
\hline Sinovac & 1065 & 75 \\
\hline Sputnik V & 381 & 19 \\
\hline CanSino & 1215 & 53 \\
\hline Unknown & 13 & 2 \\
\hline Vaccination abroad & 11 & 1 \\
\hline
\end{tabular}

Hence, the importance of conducting pharmacovigilance studies that allow the early identification of events supposedly attributable to vaccination or immunization in different groups of subjects: over 60 years, between 18 and 59 years, pregnant women and children.

Furthermore, cost-effectiveness studies provide strong evidence that vaccinating working-age adults substantially reduces SARS-CoV-2 infection. It is observed that after 2 months of introducing the BNT62b2 vaccine, it protects against symptomatic and asymptomatic infections. In addition, it could help reduce the transmission of the infection in the population. However, conclusions on prevention of transmission require more time for follow-up and analysis. Vaccination does not completely eliminate the risk of infection and therefore personal protective equipment, non-pharmaceutical interventions, and regular asymptomatic testing will be necessary until the prevalence of SARS-CoV-2 is extremely low, to reduce the risk of transmission in healthcare?

\section{Conflicts of interest}

The author declares no conflicts of interest.

\section{References}

1. Excler JL, Saville M, Berkley S, Kim JH. Vaccine development for emerging infectious diseases. Nat Med. 2021;27:591-600.

2. Declaración Para los Profesionales de la Salud: cómo se Regulan las Vacunas Contra la COVID 19 Para Garantizar que son Seguras y Eficaces. Declaración Conjunta de la Coalición Internacional de Autoridades Reguladoras de Medicamentos y la Organización Mundial de la Salud. Available from: https://www.who.int/es/news/item/11-06-2021-statement-for-healthcare-professionals-how-covid-19-vaccines-are-regulated-for-safety-and-effectiveness. [Last accessed on 2021 Jun 11].

3. NORMA Oficial Mexicana NOM-220-SSA1-2016, Instalación y Operación de la Farmacovigilancia. Diario Oficial de la Federación. Available from: http://dof.gob.mx/nota_detalle.php?codigo=5490830 and fecha. [Last accessed on 2017 Jul 19]. 
4. Secretaría de Salud. Información de la Vacuna. Available from: http:// www.vacunacovid.gob.mx/wordpress/informacion-de-la-vacuna. [Last accessed on 2021 Jun 15]

5. Gobierno de México. Conferencia Presidencial 15 de junio del 2021. Available from: https://www.gob.mx/insabi/videos/conferenciapresidencial-15-de-junio-del-2021-pulso-de-la-salud-y-vacunacion-del-presidente. [Last accessed on 2021 Jun 15].
6. Secretaría de Salud. COVID-19 México, Comunicado Técnico Diario. Available from: https://www.gob.mx/cms/uploads/attachment/file/645820/ Salud_CTD_12jun21.pdf. [Last accessed on 2021 Jun 12].

7. Hall VJ, Foulkes S, Saei A Andrews N, Oguti B, Charlett A, et al. COVID-19 vaccine coverage in health-care workers in England and effectiveness of BNT162b2 mRNA vaccine against infection (SIREN): a prospective, multicentre, cohort study. Lancet. 2021;397:1725-35. 